\title{
Pour un véritable contrôle des produits du tabac
}

\author{
Dominique Sprumonta, , Luca Crivellia, c, Sandra Nocera ${ }^{a}$, Nino Künzlia, d \\ ${ }^{a}$ Direction de la Swiss School of Public Health (SSPH+), Zurich; ${ }^{b}$ Institut de droit de la santé (IDS), Université de Neuchâtel; ${ }^{c}$ Scuola universitaria professio- \\ nale della Svizzera italiana (SUPSI), Manno, et Università della Svizzera italiana, Lugano; d Schweizerisches Tropen- und Public Health-Institut (Swiss TPH), \\ Bâle, et Université de Bâle
}

\section{Le réseau académique de l'Ecole Suisse de Santé Publique (SSPH+) rejette l'avant- projet de loi fédérale sur les produits du tabac et les cigarettes électroniques. Il est contraire à une politique basée sur les preuves scientifiques (evidence-based policy) et ne permet pas d'atteindre les objectifs qui lui sont fixés.}

En 2016, l'Assemblée fédérale a refusé d'entrer en matière sur un projet de loi fédérale sur les produits du tabac et a demandé au Conseil fédéral d'élaborer un texte qui ne porte pas atteinte à la publicité, ni aux libertés individuelles des consommateurs ${ }^{1}$. Ce faisant, nos députés ont repris sans réserve la position des cigarettiers au mépris de la santé publique et de la volonté populaire, qui souhaite, dans sa majorité, avoir une législation plus restrictive en matière de contrôle du tabac. L'administration fédérale a respecté le mandat du Parlement et a mis en consultation fin 2017 un deuxième avant-projet de loi sur les produits du tabac (AP-LPTab). Compte tenu des enjeux de santé publique, mais aussi de respect des droits fondamentaux des citoyens, qu'ils soient fumeurs ou non-fumeurs, l'Ecole Suisse de Santé Publique (SSPH+) en a fait une analyse détaillée.

Scientifiquement, le constat est sans appel: le texte proposé doit être rejeté. Dans sa conception actuelle, l'avant-projet ne permet pas d'atteindre l'objectif qui lui est fixé, à savoir «de protéger l'être humain contre les effets nocifs liés à la consommation des produits du tabac et des cigarettes électroniques» (art. 1 AP-LPTab). L'art. 4 al 2 AP-LPTab précisant que les produits «sont réputés trompeurs lorsqu'ils peuvent induire en erreur le consommateur sur les effets sur la santé, les risques ou les émissions du produit", cet avant-projet s'avère même une tromperie selon ses propres termes.

Le tabac est la première cause évitable de décès dans notre pays et dans le monde. Chaque année en Suisse, 9500 personnes meurent en raison du tabac, ce qui représente $15 \%$ des décès. De plus, 400000 personnes, dont $85 \%$ fument, souffrent d'une maladie respiratoire irréversible, la bronchopneumopathie chronique obs- tructive (BPCO). Les moyens pour agir contre cette épidémie sont pourtant connus et basés sur des preuves scientifiques. Ces standards ont du reste été traduit dans la Convention-cadre de l'OMS pour la lutte antitabac. Ils se fondent notamment sur un double constat: premièrement, la plupart des fumeurs ont commencé avant 18 ans et toute mesure qui vise à protéger les mineurs a ainsi un impact à long terme; deuxièmement, la grande majorité des fumeurs expriment un désir d'arrêter, regrettent d'avoir commencé et se considèrent comme dépendants. L'industrie du tabac partage ce constat puisqu'elle cible surtout les jeunes dans sa publicité et qu'elle manipule le contenu et la conception des cigarettes de manière à augmenter la dépendance des fumeurs.

La santé et le coût des primes d'assurance-maladie constituent une des premières préoccupations de la population. Cette thématique accapare beaucoup les débats aux Chambres fédérales. La position du Parlement en matière de produits du tabac s'avère à contre-courant par rapport à cette réalité. Les dégâts économiques du tabagisme représentent par année 1,5 milliards de francs de coûts directs liés aux soins médicaux et près de 4 milliards de pertes de productivité. Ces montants se rapprochent du chiffre d'affaire de l'industrie du tabac et dépassent largement les revenus fiscaux qu'elle génère (2,6 milliards en 2014). Du point de vue de l'économie de la santé, les mesures de prévention du tabagisme et celles encourageant l'arrêt de la fumée apportent des bénéfices conséquents en termes de santé populationnelle au regard de leurs coûts. Le rapport coût-efficacité d'une politique stricte de contrôle des produits du tabac est particulièrement positif. 
La démarche de la SSPH+ est peu courante dans le monde académique. Mais la situation est aujourd'hui exceptionnelle aussi bien sous l'angle de la santé publique que des libertés fondamentales. L'objectif de la loi devrait être de garantir les droits des non-fumeurs comme des fumeurs. Les fumeurs ne doivent pas être stigmatisés. Ils doivent pouvoir vivre leur choix de manière à ne pas interférer avec les droits des nonfumeurs, mais ils doivent aussi bénéficier des moyens adéquats pour pouvoir lutter contre leur addiction. Loin de défendre la liberté des consommateurs, comme le soutiennent les promoteurs de l'AP-LPTab, ce texte limite fortement leur faculté d'exercer leur libre choix. Il vise en réalité à protéger l'industrie du tabac et les secteurs économiques qui en dépendent, sans considération pour l'intérêt public. Cet avant-projet illustre de manière dramatique les limites de notre système de milice et l'importance des lobbys dans le débat politique. A une époque où il est toujours plus question de fake news, défendre des décisions politiques basées sur des preuves scientifiques est une nécessité. Adopter l'avant-projet de loi sur les produits du tabac serait ainsi tout aussi incompréhensible que de nier le réchauffement climatique. Nous ne pouvons ainsi qu'inviter toutes et tous, médecins, soignants, professionnels de la santé et acteurs de la santé publique, de faire passer le message auprès de leurs représentants et d'exiger une loi qui respecte les principes d'une politique basée sur les preuves scientifiques.

La consultation se termine le 23 mars 2018. L'avantprojet de loi et le commentaire sont disponibles sur: www.admin.ch/ch/f/gg/pc/pendent.html\#DFI

Vous pouvez trouver la prise de position de la SSPH+ sur son site:

www.ssphplus.ch/en/news/

\section{$\mathrm{SSPH}+$}

La SSPH+ est une fondation qui réunit huit universités. Elle englobe la faculté interuniversitaire des sciences de la santé publique de Suisse. 